biosimilar IFX switch (Remicade ${ }^{\circledR}$ to Remsima ${ }^{\circledR}$ to Zessly ${ }^{\circledR}$ ), in a real-life IBD cohort.

Methods A retrospective cohort study was carried out, and eligible patients were identified through a in-house database. All patients on treatment with $\operatorname{Remsima}^{\circledR}$ were eligible for the switch to Zessly ${ }^{\circledR}$. The primary aim of the study was to assess the clinical outcomes (in terms of continuation of therapy, loss of response and achievement of remission allowing cessation of biologic therapy) between the two patient groups, over a six month period following their switch to Zessly ${ }^{\circledR}$. Secondary outcomes measured were infusion reactions and rates of hospitalisation for an acute flare.

Results A total of 147 patients were eligible for and underwent the biosimilar-to-biosimilar switch; median age was 41 (IQR 28-56), 95 (64.6\%) were male, and 107 (72.8\%) had Crohn's Disease. Of the total cohort, 96 (65.3\%) were undergoing their first biosimilar switch (Remsima ${ }^{\circledR}$ to Zessly ${ }^{\circledR}$ ), whereas $51(34.7 \%)$ were undergoing their second biosimilar switch (Remicade ${ }^{\circledR}$ to Remsima ${ }^{\circledR}$ to Zessly ${ }^{\circledR}$ ). In those with one versus two biologic switches, there were no differences seen in the number of patients being able to continue Zessly ${ }^{\circledR}$ $(79.2 \%$ vs $80.4 \%, \mathrm{p}=0.861)$, loss of response to Zessly ${ }^{\circledR}$ $(11.5 \%$ vs $5.9 \%, p=0.273)$, or achievement of remission on Zessly ${ }^{\circledR}$ resulting in cessation of therapy $(1.0 \%$ vs $0 \%$, $\mathrm{p}=0.465)$. None of the patients from either group experienced any infusion-related reactions. There was no difference in hospitalisation in the six months following biologic switch (3.1\% vs $2.0 \%, \mathrm{p}=0.680)$.

Conclusions This real-life, single centre, short-term evaluation demonstrated that switching between biosimilar IFX brands appears to be as safe and effective as switching from originator to biosimilar IFX. A larger randomised-controlled study would be needed to confirm both the safety and effectiveness of switching between multiple biosimilar brands to substantiate the results of this evaluation.

\section{PMO-34 IBD DISK TOOL FOR EVALUATION OF PATIENT REPORTED OUTCOMES IN PATIENTS SUFFERING FROM RECURRENT POUCHITIS}

Sharafaath Shariff*, Neel Sharma, Rachel Cooney. Queen Elizabeth Hospital Birmingham, Birmingham, UK

\subsection{6/gutjnl-2021-BSG.173}

Introduction Although the primary treatment for UC is medical, approximately $20 \%$ to $30 \%$ of patients with Ulcerative Colitis (UC) will undergo proctocolectomy. (1) Ileal Pouch anal anastomosis (IPAA) surgery is an option for patients to restore gut continuity post proctocolectomy. The majority of patients will develop acute pouchitis and over time up to $25 \%$ develop chronic pouchitis. (2) The IBD-Disk was adapted from the Inflammatory Bowel Disease -Disability Index (IBDDI) as a tool to capture patient's functional status for Health Care Professionals (HCPs) to review. (3) We report the patient use and applicability of the IBD-Disk in pouchitis patients at a tertiary IBD Centre in the West Midlands.

Methods 11 patients attending the pouch clinic at a tertiary IBD centre in West Midlands were interviewed over a 6 month period (December 2020 to May 2021) with regards to their functional disability using the IBD Disk. In addition they were asked to provide their insights as to what additional symptoms should be included as part of the Disk. This element was deemed crucial as the original IBD Disk did not include patient involvement in its design.

Results 9/11 (82\%) were male. Age ranged from 35 to 73 years, mean 51 years. 8/11 (72\%) were White British and the remaining were Asian. Mean pouch duration was 18 years, range 3 to 30 years. Except for 1 patient, all others had a single IPAA procedure. 5/11 (45\%) had a single course of antibiotics with a maximum duration of a month, namely Ciprofloxacin or Metronidazole.

Out of the 10 components of the IBD Disk, lack of energy and feeling tired was the most important for our patients with a mean score of 7.3/10 followed by abdominal pain with a mean score of 6.7 and difficulty sleeping with a mean score of 6.4. The lowest scoring domains were education and work, mean score 3.5 followed by interpersonal interactions (3.7).

Additional symptoms that were important to this patient group but not included in IBD disk were mental wellbeing, rectal bleeding and fever. Incontinence for liquid stools also proved to be an important concern to $82 \%$ (9/11) of patients. Further aspects of concern comprised needing to wear a pad or plug and the effect of food on their pouch symptoms (54.5\%) (6/11).

Conclusions Currently there are no validated patient reported outcome (PRO) tools available to assess patients suffering from pouchitis. The IBD Disk was used to assess its potential in our pouchitis patients. Its limitation however is that it is not designed for pouchitis specifically and was not developed with patient involvement. This work would suggest however that IBD disk may be adaptable for use in this patient group by the addition of the following symptoms- mental well being, rectal bleeding, fever and incontinence.

\section{REFERENCES}

1. Leijonmarck CE, Persson PG, Hellers G. Factors affecting colectomy rate in ulcerative colitis: an epidemiologic study. Gut. 1990 Mar;31(3):329-33. doi: 10.1136/ gut.31.3.329. PMID: 2323599; PMCID: PMC1378277.

2. Darrell S. Pardi, MD, Geert D'Haens, MD, PhD, Bo Shen, MD, Simon Campbell, MD, Paolo Gionchetti, MD, PhD, Clinical Guidelines for the Management of Pouchitis, Inflammatory Bowel Diseases, Volume 15, Issue 9, 1 September 2009, Pages 1424-1431, https://doi.org/10.1002/ibd.21039

3. Ghosh S, Louis E, Beaugerie L, Bossuyt P, Bouguen G, Bourreille A, Ferrante $M$, Franchimont D, Frost K, Hebuterne X, Marshall JK, O'Shea C, Rosenfeld G, Williams C, Peyrin-Biroulet L. Development of the IBD Disk: A Visual Self-administered Tool for Assessing Disability in Inflammatory Bowel Diseases. Inflamm Bowe Dis. 2017 Mar;23(3):333-340. doi: 10.1097/MIB.0000000000001033. PMID: 28146002; PMCID: PMC5319390.

\section{PM0-35 UK NATIONAL AUDIT ON DIAGNOSIS AND MANAGEMENT OF COLITIS IN PATIENTS WITH PRIMARY SCLEROSING CHOLANGITIS}

${ }^{1}$ Evangelia Fatourou*, ${ }^{2}$ Dominic King, ${ }^{3}$ Sarah Hyde, ${ }^{4}$ Martine Walmsley, ${ }^{1}$ Graeme Alexander, ${ }^{2}$ Palak Trivedi, ${ }^{3}$ Simon Rushbrook, 'Douglas Thorburn, Investigating Contributors UK PSC. 'Sheila Sherlock Liver Centre and UCL Institute for Liver and Digestive Health, Royal Free Hospital, London, United Kingdon; ' University Hospitals Birmingham, Birmingham, UK; ${ }^{3}$ Department of Hepatology, Norwich Medical School, University of East Anglia, Norwich, UK; ${ }^{4}$ PSC Support, Oxford, UK

\subsection{6/gutjnl-2021-BSG.174}

Introduction The increased risk of colorectal cancer in patients with primary sclerosing cholangitis (PSC) and inflammatory bowel disease (IBD) justifies an enhanced surveillance strategy with annual colonoscopy and dye spray or protocol biopsies. As symptoms are frequently mild in PSC-IBD colitis can be missed unless colonoscopy and biopsies are undertaken at 